MATEMATIKA, 2017, Volume 33, Number 1, 55-69

(C) Penerbit UTM Press. All rights reserved

\title{
Linear program fixed-point representation
}

\author{
Jalaluddin Abdullah* \\ e-mail: jalaludn@gmail.com
}

\begin{abstract}
From a linear program and its asymmetric dual, invariant. primal and dual problems are constructed. Regular mappings are defined between the solution spaces of the original and invariant problems. The notion of centrality is introduced and subsets of regular mappings are shown to be inversely related surjections of central elements, thus representing the original problems as invariant problems. A fixed-point problem involving an idempotent symmetric matrix is constructed from the invariant problems and the notion of centrality carried over to it; the non-negative central fixed-points are shown to map one-to-one to the central solutions to the invariant problems, thus representing the invariant problems as a fixed-point problem and, by transitivity, the original problems as a fixed-point problem.
\end{abstract}

Keywords optimization; representation; linear programming; fixed-point; non-negative

2010 Mathematics Subject Classification 90C05

\section{Introduction}

The problem this paper addresses is to maximize $\boldsymbol{c}^{T} \boldsymbol{x}$ subject to $A \boldsymbol{x} \geq \boldsymbol{b}$, where $A$ is an $m \times n$ matrix, $\boldsymbol{b}$ is an $m$-dimensional vector, $\boldsymbol{c}$ is an $n$-dimensional vector, and $\boldsymbol{x}$ is an $n$-dimensional vector; The problem is written as

$$
\max \left\{\boldsymbol{c}^{T} \boldsymbol{x}: A \boldsymbol{x} \geq \boldsymbol{b}\right\}
$$

and is called the original primal linear program (LP), or simply the original primal. The aim of the paper is to represent Problem 1 as a fixed-point problem to make it more amenable to solution.

The LP and its asymmetric dual are represented as a fixed-point problem of the form $\mathfrak{P} \boldsymbol{\omega}=\boldsymbol{\omega} \geq \mathbf{0}$, where $\mathfrak{P}$ is an idempotent symmetric matrix. Preliminary notions of function pairs, regular pairs, central elements and invariance are introduced. Next the original linear program and its asymmetric dual problem are introduced and their invariant forms are constructed. Then primal and dual function pairs are introduced which map between and establish the equivalence of the original and invariant problems as well as allowing central forms of all the problems to be defined. Finally the notion of centrality is carried over to the fixed-point problem which is then shown to be equivalent to solving the central forms of the invariant problems, thus completing the representation.

The representation is related to that of Pyle [1], [2], Cline [3], Nguyen [4], Bruni et al. [5] where successive increases in simplicity and precision have occurred, particularly with the later two authors; the aim of this paper is to continue this trend.

*The author lectured at UTM and Akademi Tentera Malaysia 


\section{Preliminaries}

\subsection{The Moore-Penrose pseudo-inverse}

The development requires the Moore-Penrose pseudo-inverse: if $X$ is a matrix then there exists a unique pseudo-inverse $X^{+}$satisfying the four non-constructive conditions:

$$
\begin{array}{ll}
X X^{+} X= & X, \\
X^{+} X X^{+}= & X^{+}, \\
\left(X X^{+}\right)^{T}= & X X^{+} \text {and } \\
\left(X^{+} X\right)^{T}= & X^{+} X .
\end{array}
$$

Refer to Albert [6] for a detailed exposition.

\section{$2.2 \quad$ Function pairs}

Two functions are said to be a function pair if the domain of each is the codomain of the other.

\subsubsection{Regular pairs}

Motivated by semigroup nomenclature [7], a function pair, say $\{t, u\}$, is called a regular function pair if $t u t=t$ and $u t u=u$.

\subsubsection{Central elements}

Given a function pair $\{t, u\}$, with $t: V \rightarrow W$ and $u: W \rightarrow V$, the sets $V_{c}=W u$ and $W_{c}=V t$ are called central; elements in central sets are called central, that is

Definition 1 An element is central in the domain of one function of a function pair if the element is in the range of the other function of the pair.

Functions of the form $t_{c}=t \mid V_{c}$ with codomain $V t$, and $u_{c}=u \mid W_{c}$ with codomain $W u$ are called central functions, and function pairs of the form $\left\{t_{c}, u_{c}\right\}$ are called central function pairs.

Lemma 1 Given a regular function pair with $t: V \rightarrow W$ and $u: W \rightarrow V$,

(a) $t_{c}$ is bijective,

(b) $u_{c}$ is bijective,

(c) $V_{c}=W_{c} u ; W u=V t u$

(d) $W_{c}=V_{c} t, V t=W u t$ and

(e) $t_{c}$ and $u_{c}$ are mutual inverses.

\section{Proof}

(a) $t_{c}$ is 1:1: $\left(v_{1}, v_{2} \in W u\right) \wedge\left(v_{1} t=v_{2} t\right) \Rightarrow\left(v_{1}=w_{1} u\right) \wedge\left(v_{2}=w_{2} u\right) \wedge\left(v_{1} t=v_{2} t\right)$ $\Rightarrow\left(v_{1}=w_{1} u\right) \wedge\left(v_{2}=w_{2} u\right) \wedge\left(w_{1} u t=w_{2} u t\right) \Rightarrow\left(v_{1}=w_{1} u\right) \wedge\left(v_{2}=w_{2} u\right) \wedge\left(w_{1} u t u=\right.$ $\left.w_{2} u t u\right) \Rightarrow\left(v_{1}=w_{1} u\right) \wedge\left(v_{2}=w_{2} u\right) \wedge\left(w_{1} u=w_{2} u\right) \Rightarrow v_{1}=v_{2}$, further $t_{c}$ is onto $W_{c}:$ image $\left(t_{c}\right)=V_{c} t_{c}=V_{c} t \subseteq V t=W_{c}=V t=V t u t \subseteq W u t=V_{c} t=V_{c} t_{c}=$ image $\left(t_{c}\right)$, that is image $\left(t_{c}\right)=W_{c}$, so $t_{c}$ is bijective. 
(b) The proof is similar to (a).

(c) $V_{c}=W u=W u t u \subseteq V t u=W_{c} u \subseteq W u=V_{c}$, so $V_{c}=W_{c} u ; W u=W u t u \subseteq V t u \subseteq$ $W u$, so $W u=V t u$.

(d) the proof is similar to (c).

(e) For $v \in V_{c}, v=w u \exists w \in W \Rightarrow v t_{c} u_{c}=w u t_{c} u_{c}=w u t u_{c}=w u t u=w u=v$, so $t_{c} u_{c}$ is the identity map on $V_{c}$, similarly it is found that $u_{c} t_{c}$ is the identity map on $W_{c}$, so $t_{c}$ and $u_{c}$ are mutual inverses.

Lemma 2 Given the regular function pair $\{f, \mathfrak{f}\}$ with $f: X \rightarrow \mathfrak{X}$ and $\mathfrak{f}: \mathfrak{X} \rightarrow X$,

(a) $\boldsymbol{x}$ is central iff $\boldsymbol{x}=\boldsymbol{x} f \mathfrak{f}$.

(b) $\mathfrak{x}$ is central iff $\mathfrak{x}=\mathfrak{x} f f$.

\section{Proof}

(a) $\boldsymbol{x}$ is central $\Leftrightarrow \boldsymbol{x}=\mathfrak{x} \mathfrak{f} \exists \mathfrak{x} \in \mathfrak{X} \Rightarrow(\boldsymbol{x} f \mathfrak{f}=\mathfrak{x} \mathfrak{f} f \mathfrak{f}) \wedge(\boldsymbol{x}=\mathfrak{x} \mathfrak{f}) \exists \mathfrak{x} \in \mathfrak{X} \Rightarrow(\boldsymbol{x} f \mathfrak{f}=\mathfrak{x} \mathfrak{f}) \wedge(x=$ $\mathfrak{x} \mathfrak{x}) \exists \mathfrak{x} \in \mathfrak{X} \Rightarrow \boldsymbol{x}=\boldsymbol{x} f \mathfrak{f} \Rightarrow \boldsymbol{x}=\mathfrak{x} \mathfrak{f}$ where $\mathfrak{x}=\boldsymbol{x} f \Leftrightarrow \boldsymbol{x}$ is central, that is $\boldsymbol{x}$ is central iff $\boldsymbol{x}=\boldsymbol{x} f \mathfrak{f}$.

(b) The proof is exactly similar to that of (a).

\subsection{The invariant framework}

Quantities which are invariant under affine transformation of the solution space of Problem 1 are introduced here. These quantities are used to construct the invariant problems of Section 4.

Define

$$
\begin{aligned}
\mathfrak{A} & =A A^{+}, \\
\mathfrak{b} & =\left(I-A A^{+}\right) \boldsymbol{b}, \\
\mathfrak{c} & =A^{T+} \boldsymbol{c}, \\
\mathfrak{D} & =I-A A^{+} .
\end{aligned}
$$

Note that $\mathfrak{A}$ and $\mathfrak{D}$ are symmetric idempotents in view of the latter two non-constructive conditions for the pseudo-inverse. It is a straightforward matter, using the definitions above and the nonconstructive characterization of the Moore-Penrose pseudo-inverse, to show that

$$
\begin{array}{lll}
\mathfrak{A}^{T} & = & \mathfrak{A} \\
\mathfrak{A}^{2} & = & \mathfrak{A} \\
\mathfrak{D}^{T} & = & \mathfrak{D} \\
\mathfrak{D}^{2} & = & \mathfrak{D} \\
\mathfrak{A} \mathfrak{D}=\mathfrak{D A} & = & 0
\end{array}
$$

$$
\mid \begin{aligned}
& \mathfrak{A} \mathfrak{b}=\mathbf{0} \\
& \mathfrak{D b}=\mathfrak{b} \\
& \mathfrak{A} \mathfrak{c}=\mathfrak{c} \\
& \mathfrak{D} \mathfrak{c}=\mathbf{0}
\end{aligned}
$$

The results listed in (4) and a few obvious consequences are used throughout without necessarily referencing them.

As an intermediate step in representing the LP problem as a fixed-point problem, an invariant primal and an invariant dual are constructed, the term invariant being used as these problems can be shown to be invariant under affine transformation of the solution space of Problem 1. For details refer to [8] and [9]. 


\subsection{Quasi-boundedness}

If Problem 1 is feasible there exists a solution, say $\boldsymbol{x}_{f}$ such that $A \boldsymbol{x}_{f} \geq \boldsymbol{b}$. Now $A \boldsymbol{x}_{f} \geq$ $\boldsymbol{b} \Rightarrow A\left(x_{f}+\lambda\left(I-A^{+} A\right) \boldsymbol{c}\right) \geq \boldsymbol{b}$, so $\boldsymbol{x}_{f}+\lambda\left(I-A^{+} A\right) \boldsymbol{c}$ is also feasible and its objective value is $\boldsymbol{c}^{T}\left(x_{f}+\lambda\left(I-A^{+} A\right) \boldsymbol{c}\right)=\boldsymbol{c}^{T} x_{f}+\boldsymbol{c}^{T} \lambda\left(I-A^{+} A\right) \boldsymbol{c}=\boldsymbol{c}^{T} x_{f}+\lambda \boldsymbol{c}^{T}\left(I-A^{+} A\right) \boldsymbol{c}=$ $\boldsymbol{c}^{T} x_{f}+\lambda\left\|\left(I-A^{+} A\right) \boldsymbol{c}\right\|^{2}$, which is unbounded unless $\left\|\left(I-A^{+} A\right) \boldsymbol{c}\right\|=0$, that is unless $A^{+} A \boldsymbol{c}=\boldsymbol{c}$, so the following definition is introduced:

Definition 2 Problem 1 is said to be quasi-bounded if $A^{+} A \boldsymbol{c}=\boldsymbol{c}$.

Lemma 3 Problem 1 is feasible bounded $\Rightarrow$ Problem 1 is quasi-bounded.

Note that usually $A$ is of full rank and $m \geq n$, so $A^{+} A=I$, which implies $A^{+} A \boldsymbol{c}=\boldsymbol{c}$, and so such problem is quasi-bounded.

Lemma 4 Problem 1 is quasi-bounded $\Leftrightarrow A^{+} A \boldsymbol{c}=\boldsymbol{c} \Leftrightarrow A^{T} \mathfrak{c}=\boldsymbol{c}$.

Proof Problem 1 is quasi-bounded $\Rightarrow A^{T} \stackrel{(3 \mathrm{c})}{=} A^{T}\left(A^{T+} \boldsymbol{c}\right)=\left(A^{T} A^{T+}\right) \boldsymbol{c}$

$=\left(A^{T} A^{+T}\right) \boldsymbol{c}=\left(A^{+} A\right)^{T} \boldsymbol{c}=A^{+} A \boldsymbol{c} \stackrel{(\text { Definition } 2)}{=} \boldsymbol{c} \Rightarrow A^{T} \mathfrak{c}=\boldsymbol{c} \Rightarrow\left(A^{T} \mathbf{c}=\boldsymbol{c}\right) \wedge\left(A^{+} A A^{T} \mathbf{c}=\right.$ $\left.A^{+} A \boldsymbol{c}\right) \Rightarrow\left(A^{T} \mathfrak{c}=\boldsymbol{c}\right) \wedge\left(A^{T} \mathfrak{c}=A^{+} A \boldsymbol{c}\right) \Rightarrow A^{+} A \boldsymbol{c}=\boldsymbol{c} \stackrel{(\text { Definition } 2)}{\Rightarrow} \quad$ Problem 1 is quasibounded, so Problem 1 is quasi-bounded $\Leftrightarrow A^{T} \mathfrak{c}=\boldsymbol{c}$.

\section{The original problems}

\subsection{The primal}

The form of the LP used is the original primal given by (1), that is $\max \left\{\boldsymbol{c}^{T} \boldsymbol{x}: A \boldsymbol{x} \geq \boldsymbol{b}\right\}$.

A feasible vector is one which satisfies the constraint conditions for the linear program, for example for Problem 1, $\boldsymbol{x}$ is feasible if $A \boldsymbol{x} \geq \boldsymbol{b}$. By feasible bounded is meant the situation where the set of objective values of all feasible solutions is bounded above if the problem is one of maximization, and below if the problem is one of minimization; an "optimal" vector means a feasible vector whose objective value is equal to the maximum for the set of feasible solutions. A feasible linear program is one which has a feasible vector; a feasible bounded linear program is one with a solution.

\subsection{The asymmetric dual}

An asymmetric dual is given by Schrijver [10, p. 95]:

$$
\max \left\{\boldsymbol{c}^{T} \boldsymbol{x}: A \boldsymbol{x} \leq \boldsymbol{b}\right\}=\min \left\{\boldsymbol{b}^{T} \boldsymbol{y}: A^{T} \boldsymbol{y}=\boldsymbol{c}, \boldsymbol{y} \geq \mathbf{0}\right\}, \text { if either exists, }
$$

From this form

$$
\max \left\{(-\boldsymbol{c})^{T}(-\boldsymbol{x}): A(-\boldsymbol{x}) \leq-\boldsymbol{b}\right\}=\min \left\{(-\boldsymbol{b})^{T}(-\boldsymbol{y}): A^{T}(-\boldsymbol{y})=(-\boldsymbol{c}),-\boldsymbol{y} \geq \mathbf{0}\right\}
$$


which implies

$$
\max \left\{\boldsymbol{c}^{T} \boldsymbol{x}: A \boldsymbol{x} \geq \boldsymbol{b}\right\}=\min \left\{\boldsymbol{b}^{T} \boldsymbol{y}: A^{T} \boldsymbol{y}=\boldsymbol{c}, \boldsymbol{y} \leq \mathbf{0}\right\}
$$

We call

$$
\min \left\{\boldsymbol{b}^{T} \boldsymbol{y}: A^{T} \boldsymbol{y}=\boldsymbol{c}, \boldsymbol{y} \leq \mathbf{0}\right\}
$$

the original dual.

\section{The invariant problems}

In this section the invariant problems are introduced and it is shown how they relate to each other; in the following section these problems are related to the original primal and dual.

Substituting $\mathfrak{A}$ for $A, \mathfrak{b}$ for $\boldsymbol{b}$ and $\mathfrak{c}$ for $\boldsymbol{c}$ in (6),

$$
\max \left\{\mathfrak{c}^{T} \mathfrak{x}: \mathfrak{A} \mathfrak{x} \geq \mathfrak{b}\right\}=\min \left\{\mathfrak{b}^{T} \mathfrak{y}: \mathfrak{A} \mathfrak{y}=\mathfrak{c}, \mathfrak{y} \leq \mathbf{0}\right\}, \quad \text { if either exists. }
$$

Now $\min \left\{\mathfrak{b}^{T} \mathfrak{y}: \mathfrak{A} \mathfrak{y}=\mathfrak{c}, \mathfrak{y} \leq 0\right\}$

$$
\begin{aligned}
& =\min \left\{\mathfrak{b}^{T} \mathfrak{y}:(I-\mathfrak{D}) \mathfrak{y}=\mathfrak{c}, \mathfrak{y} \leq 0\right\} \\
& =\min \left\{\mathfrak{b}^{T} \mathfrak{y}: \mathfrak{y}-\mathfrak{D} \mathfrak{y}=\mathfrak{c}, \mathfrak{y} \leq 0\right\} \\
& =\min \left\{\mathfrak{b}^{T} \mathfrak{y}: \mathfrak{D}(-\mathfrak{y})-\mathfrak{c}=-\mathfrak{y},-\mathfrak{y} \geq 0\right\} \\
& \left.=\min \left\{\mathfrak{b}^{T}(-\mathfrak{y}): \mathfrak{D} \mathfrak{y}-\mathfrak{c}=\mathfrak{y}, \mathfrak{y} \geq 0\right\}\right) \\
& \left.=\min \left\{-\mathfrak{b}^{T} \mathfrak{y}: \mathfrak{D} \mathfrak{y}-\mathfrak{c}=\mathfrak{y}, \mathfrak{y} \geq 0\right\}\right) \\
& =-\max \left\{\mathfrak{b}^{T} \mathfrak{y}: \mathfrak{D} \mathfrak{y}-\mathfrak{c}=\mathfrak{y} \geq 0\right\}
\end{aligned}
$$

that is

$$
\min \left\{\mathfrak{b}^{T} \mathfrak{y}: \mathfrak{A} \mathfrak{y}=\mathfrak{c}, \mathfrak{y} \leq 0\right\}=-\max \left\{\mathfrak{b}^{T} \mathfrak{y}: \mathfrak{D} \mathfrak{y}-\mathfrak{c}=\mathfrak{y} \geq 0\right\}
$$

and from (8) and (9)

$$
\max \left\{\mathfrak{c}^{T} \mathfrak{x}: \mathfrak{A} \mathfrak{x} \geq \mathfrak{b}\right\}=-\max \left\{\mathfrak{b}^{T} \mathfrak{y}: \mathfrak{D} \mathfrak{y}-\mathfrak{c}=\mathfrak{y} \geq 0\right\}
$$

\section{Lemma 5}

(a) $\max \left\{\mathfrak{c}^{T} \mathfrak{x}: \mathfrak{A} \mathfrak{x}-\mathfrak{b}=\mathfrak{x} \geq 0\right\}=\max \left\{\mathfrak{c}^{T} \mathfrak{x}: \mathfrak{A} \mathfrak{x} \geq \mathfrak{b}\right\}$

(b) $\max \left\{\mathfrak{b}^{T} \mathfrak{y}: \mathfrak{D} \mathfrak{y}-\mathfrak{c}=\mathfrak{y} \geq 0\right\}=\max \left\{\mathfrak{b}^{T} \mathfrak{y}: \mathfrak{D} \mathfrak{y} \geq \mathfrak{c}\right\}$

\section{Proof}

(a) $\max \left\{\mathfrak{c}^{T} \mathfrak{x}: \mathfrak{A} \mathfrak{x}-\mathfrak{b}=\mathfrak{x} \geq 0\right\} \leq \max \left\{\mathfrak{c}^{T} \mathfrak{x}: \mathfrak{A} \mathfrak{x}-\mathfrak{b} \geq 0\right\}=\max \left\{\mathfrak{c}^{T} \mathfrak{x}: \mathfrak{A}(\mathfrak{A} \mathfrak{x}-\mathfrak{b})-\mathfrak{b}=\mathfrak{A} \mathfrak{x}-\right.$ $\mathfrak{b} \geq 0\}=\max \left\{\mathfrak{c}^{T}(\mathfrak{A} \mathfrak{x}-\mathfrak{b}): \mathfrak{A}(\mathfrak{A} \mathfrak{x}-\mathfrak{b})-\mathfrak{b}=\mathfrak{A} \mathfrak{x}-\mathfrak{b} \geq 0\right\} \leq \max \left\{\mathfrak{c}^{T} \mathfrak{x}: \mathfrak{A} \mathfrak{x}-\mathfrak{b}=\mathfrak{x} \geq 0\right\}$.

(b) The proof is analogous to proof of (a).

Using (10) and Lemma 5 (a) the central invariant problems are related as follows:

$$
\max \left\{\mathfrak{c}^{T} \mathfrak{x}: \mathfrak{A} \mathfrak{x}-\mathfrak{b}=\mathfrak{x} \geq 0\right\}+\max \left\{\mathfrak{b}^{T} \mathfrak{y}: \mathfrak{D} \mathfrak{y}-\mathfrak{c}=\mathfrak{y} \geq 0\right\}=0,
$$

and from (10) and Lemma 5 (b) the peripheral invariant problems are related thus:

$$
\max \left\{\mathfrak{c}^{T} \mathfrak{x}: \mathfrak{A} \mathfrak{x} \geq \mathfrak{b}\right\}+\max \left\{\mathfrak{b}^{T} \mathfrak{y}: \mathfrak{D} \mathfrak{y} \geq \mathfrak{c}\right\}=0 .
$$


The four problems in Lemma 5 comprise the invariant problems; the form in (10), on the LHS, without an equals, is called "peripheral" and the form, on the RHS, with an equals is called "central"; the problems are classified in Table 1. Later, regular function pairs which map between the original and invariant forms are introduced which justify the "central" appellation and permit the definition of peripheral and central forms of the original problems. Note (a) referring forward, Theorem 1 is the motivation for calling the invariant problems of Table 1 "central". (b) in view of Lemma 5 the central invariant problems are very similar to the peripheral invariant problems, however precise analysis of the LP problem requires focus on the central invariant problems.

Table 1: Invariant problem classification

\begin{tabular}{lll}
\hline & primal & dual \\
\hline peripheral & $\max \left\{\mathfrak{c}^{T} \mathfrak{x}: \mathfrak{A} \mathfrak{x} \geq \mathfrak{b}\right\}$ & $\max \left\{\mathfrak{b}^{T} \mathfrak{y}: \mathfrak{D} \mathfrak{y} \geq \mathfrak{c}\right\}$ \\
central & $\max \left\{\mathfrak{c}^{T} \mathfrak{x}: \mathfrak{A} \mathfrak{x}-\mathfrak{b}=\mathfrak{x} \geq 0\right\}$ & $\max \left\{\mathfrak{b}^{T} \mathfrak{y}: \mathfrak{D} \mathfrak{y}-\mathfrak{c}=\mathfrak{y} \geq 0\right\}$ \\
\hline
\end{tabular}

\section{Original and invariant problem relationship}

\subsection{The primal and dual function pairs}

With solution sets labeled as:

$X$ for the original primal (a copy of $\Re^{n}$ )

$Y$ for the original dual (a copy of $\Re^{m}$ )

$\mathfrak{X}$ for the invariant primal (a copy of $\Re^{m}$ ), and

$\mathfrak{Y}$ for the invariant dual (a copy of $\Re^{m}$ ),

the original and invariant problems are related using two function pairs as follows:

the primal function pair $\left\{f_{p}, \mathfrak{f}_{p}\right\}$ where

$$
\begin{aligned}
f_{p}: X \rightarrow \mathfrak{X}, \boldsymbol{x} \mapsto A \boldsymbol{x}-\boldsymbol{b} \text { and } \\
\mathfrak{f}_{p}: \mathfrak{X} \rightarrow X, \mathfrak{x} \mapsto A^{+}(\mathfrak{x}+\boldsymbol{b})
\end{aligned}
$$

and the dual function pair $\left\{f_{d}, \mathfrak{f}_{d}\right\}$ where

$$
\begin{aligned}
f_{d}: Y \rightarrow \mathfrak{Y}, \boldsymbol{y} \mapsto-\mathfrak{D} \boldsymbol{y}-\mathfrak{c} \text { and } \\
\mathfrak{f}_{d}: \mathfrak{Y} \rightarrow Y, \mathfrak{y} \mapsto \mathfrak{c}-\mathfrak{D} \mathfrak{y}
\end{aligned}
$$

For the primal mappings the compositions $\boldsymbol{x} f_{p} \mathfrak{f}_{p} \stackrel{(13)}{=}(A \boldsymbol{x}-\boldsymbol{b}) \mathfrak{f}_{p}$

$\stackrel{(14)}{=} A^{+}((A \boldsymbol{x}-\boldsymbol{b})+\boldsymbol{b})=A^{+} A \boldsymbol{x}$, and $\mathfrak{x} \mathfrak{f}_{p} f_{p}=\left(A^{+}(\mathfrak{x}+\boldsymbol{b})\right) f_{p}=A\left(A^{+}(\mathfrak{x}+b)\right)-\boldsymbol{b}=$ $A A^{+}(\mathfrak{x}+\boldsymbol{b})-\boldsymbol{b}=\mathfrak{A} \mathfrak{x}+\mathfrak{A} \boldsymbol{b}-\boldsymbol{b}=\mathfrak{A} \mathfrak{x}-\mathfrak{b}$ are computed, that is

$$
\begin{aligned}
\boldsymbol{x} f_{p} \mathfrak{f}_{p} & =A^{+} A \boldsymbol{x} \\
\mathfrak{x} \mathfrak{f}_{p} f_{p} & =\mathfrak{A} \mathfrak{x}-\mathfrak{b}
\end{aligned}
$$


and for the dual mappings the compositions $\boldsymbol{y} f_{d} \mathfrak{f}_{d}=(-\mathfrak{D} \boldsymbol{y}-\mathfrak{c}) \mathfrak{f}_{d}=\mathfrak{c}-\mathfrak{D}(-\mathfrak{D} \boldsymbol{y}-\mathfrak{c})=$ $\mathfrak{c}-(-\mathfrak{D} \boldsymbol{y})=\mathfrak{c}+\mathfrak{D} \boldsymbol{y}$, and $\mathfrak{y} \mathfrak{f}_{d} f_{d}=(\mathfrak{c}-\mathfrak{D} \mathfrak{y}) f_{d}=-\mathfrak{D}(\mathfrak{c}-\mathfrak{D} \mathfrak{y})-\mathfrak{c}=\mathfrak{D} \mathfrak{y}-\mathfrak{c}$ are computed, that is

$$
\begin{aligned}
\boldsymbol{y} f_{d} \mathfrak{f}_{d} & =\mathfrak{D} \boldsymbol{y}+\mathfrak{c} \\
\mathfrak{y} \mathfrak{f}_{d} f_{d} & =\mathfrak{D} \mathfrak{y}-\mathfrak{c}
\end{aligned}
$$

The central sets and optimal central subsets w.r.t. these function pairs for the original and invariant problems are indicated in Figure 1 by the black and green boxes respectively.

For the primal mappings the triple compositions

$$
\boldsymbol{x} f_{p} \mathfrak{f}_{p} f_{p} \stackrel{(17)}{=}\left(A^{+} A \boldsymbol{x}\right) f_{p} \stackrel{(13)}{=} A\left(A^{+} A \boldsymbol{x}\right)-\boldsymbol{b}=A \boldsymbol{x}-\boldsymbol{b} \stackrel{(13)}{=} \boldsymbol{x} f_{p},
$$

and

$$
\mathfrak{x} \mathfrak{f}_{p} f_{p} \mathfrak{f}_{p} \stackrel{(18)}{=}(\mathfrak{A} \mathfrak{x}-\mathfrak{b}) \mathfrak{f}_{p} \stackrel{(14)}{=} A^{+}((\mathfrak{A} \mathfrak{x}-\mathfrak{b})+\boldsymbol{b})=A^{+}(\mathfrak{A} \mathfrak{x}+\boldsymbol{b})=A^{+}(\mathfrak{x}+\boldsymbol{b}) \stackrel{(14)}{=} \quad \mathfrak{x} \mathfrak{x}_{p}
$$

are computed, that is

$$
\begin{aligned}
f_{p} \mathfrak{f}_{p} f_{p} & =f_{p} \\
\mathfrak{f}_{p} f_{p} \mathfrak{f}_{p} & =\mathfrak{f}_{p}
\end{aligned}
$$

and for the dual mappings the triple compositions

$\boldsymbol{y} f_{d} \mathfrak{f}_{d} f_{d} \stackrel{(19)}{=}(\mathfrak{D} \boldsymbol{y}+\mathfrak{c}) f_{d} \stackrel{(15)}{=}-\mathfrak{D}(\mathfrak{D} \boldsymbol{y}+\mathfrak{c})-\mathfrak{c}=-\mathfrak{D} \boldsymbol{y}-\mathfrak{c} \stackrel{(15)}{=} \boldsymbol{y} f_{d}$, and

$\mathfrak{y} \mathfrak{f}_{d} f_{d} \mathfrak{f}_{d} \stackrel{(20)}{=}(\mathfrak{D} \mathfrak{y}-\mathfrak{c}) \mathfrak{f}_{d} \stackrel{(16)}{=} \mathfrak{c}-\mathfrak{D}(\mathfrak{D} \mathfrak{y}-\mathfrak{c})=\mathfrak{c}-\mathfrak{D} \mathfrak{y} \stackrel{(16)}{=} \quad \mathfrak{y} \mathfrak{f}_{d}$ are computed, that is

$$
\begin{aligned}
f_{d} \mathfrak{f}_{d} f_{d} & =f_{d} \\
\mathfrak{f}_{d} f_{d} \mathfrak{f}_{d} & =\mathfrak{f}_{d}
\end{aligned}
$$

From (21), (22), (23) and (24), the function pairs $\left\{f_{p}, \mathfrak{f}_{p}\right\}$ and $\left\{f_{d}, \mathfrak{f}_{d}\right\}$ are regular so Lemma 1 applies, giving in the context of these functions:

\section{Theorem 1}

(a) The four central mappings $f_{p c}, \mathfrak{f}_{p c}, f_{d c}$ and $\mathfrak{f}_{d c}$ are bijective.

(b) The primal and dual central function pairs $\left\{f_{p c}, \mathfrak{f}_{p c}\right\}$ and $\left\{f_{d c}, \mathfrak{f}_{d c}\right\}$ comprise mutually inverse functions.

(c) Conditions $\boldsymbol{x}=A^{+} A \boldsymbol{x}, \boldsymbol{y}=\mathfrak{D} \boldsymbol{y}+\mathfrak{c}, \mathfrak{x}=\mathfrak{A} \mathfrak{x}-\mathfrak{b}$, and $\mathfrak{y}=\mathfrak{D} \mathfrak{y}-\mathfrak{c}$ define precisely the central elements of $X, Y, \mathfrak{X}$, and $\mathfrak{Y}$ respectively.

Proof (a) and (b) follow since the function pairs $\left\{f_{p}, \mathfrak{f}_{p}\right\}$ and $\left\{f_{d}, \mathfrak{f}_{d}\right\}$ satisfy the conditions for Lemma 1. (c) Follows from Lemma 2, and from (17), (18), (19) and (20) respectively.

\section{Theorem 2}

(a) $\boldsymbol{x}=A^{+} A \boldsymbol{x}$,

(b) $\boldsymbol{y}=\mathfrak{D} \boldsymbol{y}+\mathfrak{c}$,

(c) $\mathfrak{x}=\mathfrak{A} \mathfrak{x}-\mathfrak{b}$, and

(d) $\mathfrak{y}=\mathfrak{D} \mathfrak{y}-\mathfrak{c}$ define precisely the central elements of $X, Y, \mathfrak{X}$, and $\mathfrak{Y}$ respectively. 
Proof The theorem follows from Lemma 2, and from (17), (18), (19) and (20) respectively.

Four linear programs are now considered: the original primal (1), original dual (7), invariant primal (mentioned in Lemma $5 \mathrm{a}$ ) and invariant dual (mentioned in Lemma $5 \mathrm{~b}$ ); the original and invariant programs are related using the primal and dual function pairs defined in Section 5.1.

\subsection{Feasibility}

The quantities $A \boldsymbol{x}-\boldsymbol{b}, \boldsymbol{y}, \mathfrak{A} \mathfrak{x}-\mathfrak{b}$ and $\mathfrak{D} \mathfrak{y}-\mathfrak{c}$ are related by the defined function pairs as follows:

\section{Lemma 6}
(a) $\mathfrak{A}\left(\boldsymbol{x} f_{p}\right)-\mathfrak{b}$
$=A x-b$
(b) $A\left(\mathfrak{x} \mathfrak{f}_{p}\right)-\boldsymbol{b}$
$=\mathfrak{A} \mathfrak{x}-\mathfrak{b}$
(c) $\mathfrak{D}\left(\boldsymbol{y} f_{d}\right)-\mathfrak{c}$
$=\quad-\boldsymbol{y}$ if $A^{T} \boldsymbol{y}=\boldsymbol{c}$
(d) $A^{T}\left(\mathfrak{y} \mathfrak{f}_{d}\right)=\boldsymbol{c} \wedge \mathfrak{y} \mathfrak{f}_{d}=-(\mathfrak{D} \mathfrak{y}-\mathfrak{c})$ if Problem 1 is quasi-bounded

\section{Proof}

(a) $\mathfrak{A}\left(\boldsymbol{x} f_{p}\right)-\mathfrak{b} \stackrel{(18)}{=}\left(\boldsymbol{x} f_{p}\right) \mathfrak{f}_{p} f_{p}=\boldsymbol{x}\left(f_{p} \mathfrak{f}_{p} f_{p}\right) \stackrel{(21)}{=} \boldsymbol{x} f_{p} \stackrel{(13)}{=} A \boldsymbol{x}-\boldsymbol{b}$,

(b) $A\left(\mathfrak{x} \mathfrak{f}_{p}\right)-\boldsymbol{b} \stackrel{(13)}{=}\left(\mathfrak{x} \mathfrak{f}_{p}\right) f_{p} \stackrel{(18)}{=} \mathfrak{A} \mathfrak{x}-\mathfrak{b}$

(c) $\mathfrak{D}\left(\boldsymbol{y} f_{d}\right)-\mathfrak{c} \stackrel{(20)}{=} \boldsymbol{y} f_{d}\left(\mathfrak{f}_{d} f_{d}\right)=\boldsymbol{y}\left(f_{d} \mathfrak{f}_{d} f_{d}\right) \stackrel{(23)}{=} \boldsymbol{y} f_{d} \stackrel{(15)}{=}-\mathfrak{D} \boldsymbol{y}-\mathfrak{c}=-(I-\mathfrak{A}) \boldsymbol{y}-\mathfrak{c}=$ $-\boldsymbol{y}+A A^{+} \boldsymbol{y}-\mathfrak{c}=-\boldsymbol{y}+A^{+T} A^{T} \boldsymbol{y}-\mathfrak{c}=-\boldsymbol{y}+A^{+T} \boldsymbol{c}-\mathfrak{c}=-\boldsymbol{y}+\mathfrak{c}-\mathfrak{c}=-\boldsymbol{y}$,

(d) $\mathfrak{y} \tilde{f}_{d}=\mathfrak{c}-\mathfrak{D} \mathfrak{y}=-(\mathfrak{D} \mathfrak{y}-\mathfrak{c})$, so $A^{T}\left(\mathfrak{y} \mathfrak{f}_{d}\right)=A^{T}(\mathfrak{c}-\mathfrak{D} \mathfrak{y})=A^{T}\left(\mathfrak{c}-\left(I-A A^{+}\right) \mathfrak{y}\right)=$ $A^{T} \mathfrak{c} \stackrel{(\operatorname{Lemma} 4 c)}{=} \boldsymbol{c}$, .

From Lemma 6 it is seen that feasible solutions are mapped to feasible solutions:

\section{Corollary 1}

(a) $A \boldsymbol{x} \geq \boldsymbol{b} \Leftrightarrow \mathfrak{A}\left(\boldsymbol{x} f_{p}\right) \geq \mathfrak{b}$,

(b) $\mathfrak{A} \mathfrak{x} \geq \mathfrak{b} \quad \Leftrightarrow A\left(\mathfrak{x} \mathfrak{f}_{p}\right) \geq \boldsymbol{b}$,

(c) $\boldsymbol{y} \leq 0 \Leftrightarrow \mathfrak{D}\left(y f_{d}\right) \geq \mathfrak{c}$,

if $A^{T} \boldsymbol{y}=\boldsymbol{c}$,

(d) $\mathfrak{D} \mathfrak{y} \geq \mathfrak{c} \quad \Leftrightarrow \quad A^{T}\left(\mathfrak{y} \mathfrak{f}_{d}\right)=\boldsymbol{c} \wedge \mathfrak{y} \mathfrak{f}_{d} \leq 0$, if Problem 1 is quasi-bounded.

Corollary 1 shows that the two functions of the primal function pair map between feasible original primal solutions and feasible invariant primal solutions, while the two functions of the dual function pair map between feasible original dual solutions and feasible invariant dual solutions.

\subsection{Optimality}

It is shown that the objective values of corresponding primal vectors differ by a fixed amount, and that the objective values of corresponding dual vectors move in lockstep - that is they differ in sign and by a fixed amount, and thus solutions are mapped to solutions: 


\section{Lemma 7}

(a) $\mathfrak{c}^{T}\left(\boldsymbol{x} f_{p}\right)=\boldsymbol{c}^{T} \boldsymbol{x}-\boldsymbol{b}^{T} \mathfrak{c}$

(b) $\boldsymbol{c}^{T}\left(\mathfrak{x}_{p}\right)=\mathfrak{c}_{p}^{T} \mathfrak{x}+\boldsymbol{b}^{T} \mathfrak{c}$

(c) $\mathfrak{b}^{T}\left(y f_{d}\right)=\boldsymbol{b}^{T} \mathfrak{c}-\boldsymbol{b}^{T} \boldsymbol{y}$ if $A^{T} \boldsymbol{y}=\boldsymbol{c}$

(d) $\boldsymbol{b}^{T}\left(\mathfrak{y} \mathfrak{f}_{d}\right)=\boldsymbol{b}^{T} \mathfrak{c}-\mathfrak{b}^{T} \mathfrak{y}$

\section{Proof}

(a) $\mathfrak{c}^{T}\left(\boldsymbol{x} f_{p}\right)=\mathfrak{c}^{T}(A \boldsymbol{x}-\boldsymbol{b})=\mathfrak{c}^{T} A \boldsymbol{x}-\mathfrak{c}^{T} b=\left(A^{T+} \boldsymbol{c}\right)^{T} A \boldsymbol{x}-\mathfrak{c}^{T} b=\boldsymbol{c}^{T} A^{+} A \boldsymbol{x}-\mathfrak{c}^{T} b=$ $\left(A^{+} A \boldsymbol{c}\right)^{T} x-\mathfrak{c}^{T} b \stackrel{(\text { Definition } 2)}{=} \boldsymbol{c}^{T} x-\mathfrak{c}^{T} b=\boldsymbol{c}^{T} x-\boldsymbol{b}^{T} \mathfrak{c}$

(b) $\boldsymbol{c}^{T}\left(\mathfrak{x} \mathfrak{x}_{p}\right)=\boldsymbol{c}^{T} A^{+}(\mathfrak{x}+b)=\left(A^{+T} \boldsymbol{c}\right)^{T}(\mathfrak{x}+b)=\left(A^{T+} \boldsymbol{c}\right)^{T}(\mathfrak{x}+b) \stackrel{(3 \mathrm{c})}{=} \mathfrak{c}^{T}(\mathfrak{x}+b)=\mathfrak{c}^{T} \mathfrak{x}+$ $\mathfrak{c}^{T} b=\mathfrak{c}^{T} \mathfrak{x}+b^{T} \mathfrak{c}$

(c) $\mathfrak{b}^{T}\left(y f_{d}\right)=\mathfrak{b}^{T}(-\mathfrak{D} \boldsymbol{y}-\mathfrak{c})=-\mathfrak{b}^{T} \mathfrak{D} \boldsymbol{y}=\boldsymbol{b}^{T}\left(A A^{+}-I\right) \boldsymbol{y}=\boldsymbol{b}^{T}\left(A A^{+}\right) \boldsymbol{y}-\boldsymbol{b}^{T} y=\boldsymbol{b}^{T} A^{+T} A^{T} \boldsymbol{y}-$ $\boldsymbol{b}^{T} y=\boldsymbol{b}^{T} A^{+T} \boldsymbol{c}-\boldsymbol{b}^{T} y=\boldsymbol{b}^{T} \boldsymbol{c}-\boldsymbol{b}^{T} y$

(d) $\boldsymbol{b}^{T}\left(\mathfrak{y} \mathfrak{f}_{d}\right)=\boldsymbol{b}^{T}(\mathfrak{c}-\mathfrak{D} \mathfrak{y})=\boldsymbol{b}^{T} \mathfrak{c}-\boldsymbol{b}^{T} \mathfrak{D} \mathfrak{y}=\boldsymbol{b}^{T} \mathfrak{c}-\mathfrak{b}^{T} \mathfrak{y}$.

\section{Corollary 2}

(a) $\boldsymbol{c}^{T}\left(\boldsymbol{x} f_{p}\right)+\mathfrak{b}^{T}\left(y f_{d}\right)=\boldsymbol{c}^{T} x-\boldsymbol{b}^{T} y \quad$ if $A^{T} \boldsymbol{y}=\boldsymbol{c}$

(b) $\boldsymbol{c}^{T}\left(\mathfrak{x} \mathfrak{x}_{p}\right)-\boldsymbol{b}^{T}\left(\mathfrak{y} \mathfrak{f}_{d}\right)=\mathfrak{c}^{T} \mathfrak{x}+\mathfrak{b}^{T} \mathfrak{y}$.

\section{Proof}

(a) Adding the results in Lemma 7 a and $7 \mathrm{c}, \boldsymbol{c}^{T}\left(\boldsymbol{x} f_{p}\right)+\mathfrak{b}^{T}\left(\boldsymbol{y} f_{d}\right)=\boldsymbol{c}^{T} x-\boldsymbol{b}^{T} y$ is obtained.

(b) Subtracting Lemma $7 \mathrm{~d}$ from $7 \mathrm{~b}$ yields $\boldsymbol{c}^{T}\left(\mathfrak{x} \mathfrak{x}_{p}\right)-\boldsymbol{b}^{T}\left(\mathfrak{y} \mathfrak{y}_{d}\right)=\mathfrak{c}^{T} \mathfrak{x}+\mathfrak{b}^{T} \mathfrak{y}$.

From Corollaries 1 and 2 it is seen that solutions to the original primal are mapped to solutions to the invariant primal and vice versa, and solutions to the original dual are mapped to solutions to the invariant dual and vice versa, making the representation of the primal problems as invariant problems explicit:

\section{Theorem 3}

Provided the original primal is quasi-bounded,

(a) if $\boldsymbol{x}_{s}$ and $\boldsymbol{y}_{s}$ are $\langle$ feasible|solutions $\rangle$ for the original primal and dual respectively then $\boldsymbol{x}_{s} f_{p}$ and $\boldsymbol{y}_{s} f_{d}$ are central 〈feasible|solutions〉 for the invariant primal and dual respectively.

(b) if $\mathfrak{x}_{s}$ and $\mathfrak{y}_{s}$ are 〈feasible|solutions $\rangle$ for the invariant primal and dual respectively then $\mathfrak{x}_{s} \mathfrak{f}_{p}$ and $\mathfrak{y}_{s} \mathfrak{f}_{d}$ are central $\langle$ feasible|solutions〉 for the original primal and dual respectively,

(c) The four mappings, restricted to central [feasible|solution] vectors are bijections.

\section{Proof}

(a) If $\boldsymbol{x}_{s}$ and $\boldsymbol{y}_{s}$ are feasible then, from Corollary 1 (a) and (c), $\boldsymbol{x}_{s} f_{p}$ and $\boldsymbol{y}_{s} f_{d}$ are feasible, while centrality follows from Definition 1; further, given solutions $\boldsymbol{x}_{s}$ and $\boldsymbol{y}_{s}$,

$$
\boldsymbol{c}^{T} \boldsymbol{x}_{s}-\boldsymbol{b}^{T} \boldsymbol{y}_{s}=0 \stackrel{(\text { Corollary 2a) }}{\Rightarrow} \boldsymbol{c}^{T}\left(\boldsymbol{x}_{s} f_{p}\right)+\mathfrak{b}^{T}\left(\boldsymbol{y}_{s} f_{d}\right)=0 \stackrel{(12)}{\Rightarrow} \boldsymbol{x}_{s} f_{p}
$$


and $\boldsymbol{y}_{s} f_{d}$ are optimal, and central since they are in the ranges of $f_{p}$ and $f_{d}$ respectively, and are therefor respective solutions for the invariant primal and dual.

(b) If $\mathfrak{x}_{s}$ and $\mathfrak{y}_{s}$ are feasible then, from Corollary 1 (b) and (d), $\mathfrak{x}_{s} \mathfrak{f}_{p}$ and $\mathfrak{y}_{s} \mathfrak{f}_{d}$ are feasible while centrality follows from Definition 1; further, if $\mathfrak{x}_{s}$ and $\mathfrak{y}_{s}$ are solutions then

$$
\mathfrak{c}^{T} \mathfrak{x}_{s}+\mathfrak{b}^{T} \mathfrak{y}_{s}=0 \stackrel{(\text { Corollary } 2 \mathrm{~b})}{\Rightarrow} \boldsymbol{c}^{T}\left(\mathfrak{x}_{s} \mathfrak{f}_{p}\right)-\boldsymbol{b}^{T}\left(\mathfrak{y}_{s} \mathfrak{f}_{d}\right)=0 \stackrel{(6)}{\Rightarrow} \mathfrak{x}_{s} \mathfrak{f}_{p}
$$

and $\mathfrak{y}_{s} \mathfrak{f}_{d}$ are respective solutions for the original primal and dual.

(c) For central vectors the result follows from Lemma 1; for central feasible vectors the result follows from Corollary 1 (b) and (d); for central solutions the result follows from Corollary 1 (b) and (d), and Corollary 2.

Remark 1 From Lemmas 1 and 3, the central function pairs establish a one-to-one correspondence between the central solutions to the original problems and the central solutions to the invariant problems, and a one-to-one correspondence between the central solutions to the original problems and the central solutions to the invariant problems.

The representation of the original problems as invariant problems is summarized by the lower two-thirds of Figure 1. Feasible conditions and solutions are in thin-lined boxes, central feasible conditions and solutions are in thick-lined boxes, and optimal central feasible conditions and solutions are in green boxes; the two function pairs and their central forms are shown linking the original and invariant solutions; the two functions $\mathcal{F}_{p}=\mathfrak{f}_{p} f_{p}$ and $\mathcal{F}_{d}=\mathfrak{f}_{d} f_{d}$ which map arbitrary invariant solutions to central invariant solutions are shown.

\subsection{Summary}

Invariant problems have been constructed and mappings have been defined which establish a 1:1 relationship between the solutions to the central original and central invariant problems.

\section{$6 \quad$ Invariant and fixed-point problem relationship}

A fixed-point problem is constructed which involves finding a non-negative vector

$$
\omega=\left[\begin{array}{l}
\xi \\
\zeta
\end{array}\right]
$$

which is transformed to itself by an idempotent symmetric matrix $\mathfrak{P}$. The notion of centrality is introduced to elements in the domain of $\mathfrak{N}$ via the backmap of the natural mapping

$$
\mathfrak{N}: \boldsymbol{\omega}=\left[\begin{array}{l}
\boldsymbol{\xi} \\
\boldsymbol{\zeta}
\end{array}\right] \in \Re^{2 m} \mapsto(\boldsymbol{\xi}, \boldsymbol{\zeta}), \boldsymbol{\xi} \in \Re^{m}, \boldsymbol{\zeta} \in \Re^{m}
$$

(where $\boldsymbol{\xi}$ and $\boldsymbol{\zeta}$ are interpreted as being vectors in the solution spaces of the invariant primal and dual respectively). It is then shown that $\mathfrak{N}$ with domain restricted to central solutions is a surjection onto the set of central invariant solutions, thus completing the representation of the LP problem as a fixed-point problem. 


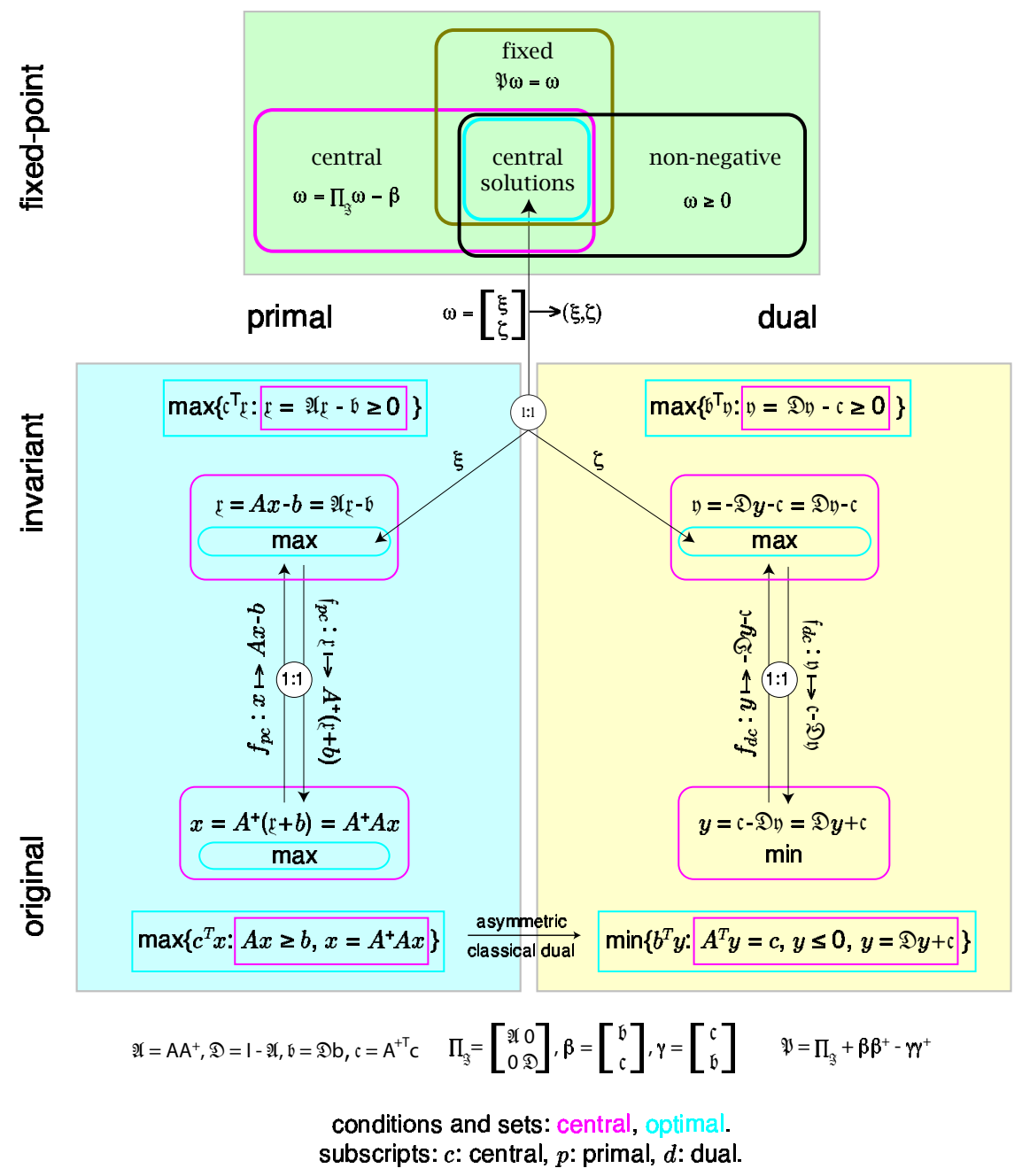

Figure 1: Original, invariant and fixed-point problem relationship 


\subsection{The fixed-point matrix}

The following notation is introduced:

$$
\begin{array}{lll}
\Pi_{\mathfrak{Z}} & =\left[\begin{array}{cc}
\mathfrak{A} & 0 \\
0 & \mathfrak{D}
\end{array}\right] \\
\boldsymbol{\beta} & =\left[\begin{array}{l}
\mathfrak{b} \\
\mathfrak{c}
\end{array}\right] \\
\gamma & =\left[\begin{array}{l}
\mathfrak{c} \\
\mathfrak{b}
\end{array}\right] \\
\Pi_{\beta} & =\boldsymbol{\beta} \boldsymbol{\beta}^{+} \\
\Pi_{\gamma} & =\boldsymbol{\gamma} \gamma^{+}
\end{array}
$$

then

$$
\begin{aligned}
& \Pi_{\mathfrak{Z}}^{2}=\Pi_{\mathfrak{Z}}^{T}=\Pi_{\mathfrak{Z}}(\mathrm{a}) \mid \Pi_{\beta} \Pi_{\gamma}=\Pi_{\gamma} \Pi_{\beta}=0 \quad(\mathrm{~g}) \\
& \Pi_{\mathfrak{Z}} \Pi_{\beta}=0 \text { (b) } \Pi_{\beta} \boldsymbol{\beta} \quad=\boldsymbol{\beta}(\mathrm{h}) \\
& \Pi_{3} \Pi_{\gamma}=\Pi_{\gamma}(\mathrm{c}) \Pi_{\beta} \gamma \quad=0 \text { (i) } \\
& \Pi_{\mathfrak{Z}} \boldsymbol{\beta}=0 \text { (d) } \Pi_{\gamma}^{2}=\Pi_{\gamma}^{T}=\Pi_{\gamma}(\mathrm{j}) \\
& \Pi_{\mathfrak{Z}} \gamma=\gamma(\mathrm{e}) \Pi_{\gamma} \boldsymbol{\beta}=0 \quad(\mathrm{k}) \\
& \Pi_{\beta}^{2}=\Pi_{\beta}^{T}=\Pi_{\beta} \text { (f) } \mid \Pi_{\gamma} \gamma=\gamma
\end{aligned}
$$

Definition 3 The fixed-point matrix is defined as $\mathfrak{P}=\Pi_{\mathfrak{Z}}+\Pi_{\beta}-\Pi_{\gamma}$, that is

$$
\mathfrak{P}=\left[\begin{array}{cc}
\mathfrak{A} & 0 \\
0 & \mathfrak{D}
\end{array}\right]+\left[\begin{array}{l}
\mathfrak{b} \\
\mathfrak{c}
\end{array}\right]\left[\begin{array}{l}
\mathfrak{b} \\
\mathfrak{c}
\end{array}\right]^{+}-\left[\begin{array}{l}
\mathfrak{c} \\
\mathfrak{b}
\end{array}\right]\left[\begin{array}{l}
\mathfrak{c} \\
\mathfrak{b}
\end{array}\right]^{+}
$$

Symmetry of $\mathfrak{P}$ follows from the symmetry of its components $\Pi_{\mathfrak{Z}}, \Pi_{\beta}$ and $\Pi_{\gamma}$, while $\mathfrak{P}^{2} \stackrel{(3)}{=}$

$\left(\Pi_{\mathfrak{Z}}+\Pi_{\beta}-\Pi_{\gamma}\right)^{2}=\Pi_{\mathfrak{Z}}^{2}+\Pi_{\mathfrak{Z}} \Pi_{\beta}-\Pi_{\mathfrak{Z}} \Pi_{\gamma}+\Pi_{\beta} \Pi_{\mathfrak{Z}}+\Pi_{\beta}^{2}-\Pi_{\beta} \Pi_{\gamma}-\Pi_{\gamma} \Pi_{\mathfrak{Z}}-\Pi_{\gamma} \Pi_{\beta}+\Pi_{\gamma}^{2} \stackrel{(26)}{=} \Pi_{\mathfrak{Z}}+$ $0-\Pi_{\gamma}+0+\Pi_{\beta}-0-\Pi_{\gamma}-0+\Pi_{\gamma}=\Pi_{\mathfrak{Z}}+\Pi_{\beta}-\Pi_{\gamma} \stackrel{(3)}{=} \mathfrak{P}$, that is

$$
\mathfrak{P}^{T}=\mathfrak{P} \text { and } \mathfrak{P}^{2}=\mathfrak{P},
$$

in other words $\mathfrak{P}$ is a projection matrix.

Further, $\mathfrak{P} \boldsymbol{\beta} \stackrel{(3)}{=}\left(\Pi_{\mathfrak{Z}}+\Pi_{\beta}-\Pi_{\gamma}\right) \boldsymbol{\beta}=\Pi_{\mathfrak{Z}} \boldsymbol{\beta}+\Pi_{\beta} \boldsymbol{\beta}-\Pi_{\gamma} \boldsymbol{\beta} \stackrel{(26)}{=} 0+\boldsymbol{\beta}-0=\boldsymbol{\beta}$, and $\mathfrak{P} \gamma \stackrel{(3)}{=}\left(\Pi_{\mathfrak{Z}}+\Pi_{\beta}-\Pi_{\gamma}\right) \gamma=\Pi_{\mathfrak{Z}} \gamma+\Pi_{\beta} \gamma-\Pi_{\gamma} \gamma \stackrel{(26)}{=} \gamma+0-\gamma=0$, that is

$$
\begin{aligned}
& \mathfrak{P} \boldsymbol{\beta}=\boldsymbol{\beta} \\
& \mathfrak{P} \boldsymbol{\gamma}=\mathbf{0} .
\end{aligned}
$$

and, obviously,

$$
\begin{aligned}
& \boldsymbol{\beta}^{T} \mathfrak{P}=\boldsymbol{\beta}^{T}, \\
& \boldsymbol{\gamma}^{T} \mathfrak{P}=\mathbf{0}^{T} .
\end{aligned}
$$


Definition 4 A point $\boldsymbol{\omega}$ is said to be a fixed-point, if $\mathfrak{P} \boldsymbol{\omega}=\boldsymbol{\omega}$.

Remark 2 It follows that for fixed-point $\omega$,

$$
\boldsymbol{\gamma}^{T} \boldsymbol{\omega}=0, \Pi_{\gamma} \boldsymbol{\omega}=\mathbf{0}, \text { and } \mathfrak{P} \boldsymbol{\omega}=\left(\Pi_{\mathfrak{Z}}+\Pi_{\beta}\right) \boldsymbol{\omega} .
$$

\subsection{The surjection}

\subsubsection{Point classification}

Attributes are ascribed to pairs as follows: $(\boldsymbol{\xi}, \boldsymbol{\zeta})$ inherits a particular characteristic iff $\boldsymbol{\xi}$ and $\boldsymbol{\zeta}$ both have this characteristic. Attributes are ascribed to points as follows: $\left[\begin{array}{l}\boldsymbol{\xi} \\ \boldsymbol{\zeta}\end{array}\right]$ inherits a particular characteristic iff $(\boldsymbol{\xi}, \boldsymbol{\zeta})$ has this characteristic so, for example, given

Definition $5(\boldsymbol{\xi}, \boldsymbol{\zeta})$ is quasi-optimal iff $\mathfrak{c}^{T} \boldsymbol{\xi}+\mathfrak{b}^{T} \boldsymbol{\zeta}=0$.

If $\left(\boldsymbol{\xi}_{q}, \boldsymbol{\zeta}_{q}\right)$ is quasi-optimal then, by ascription, $\left[\begin{array}{l}\boldsymbol{\xi}_{q} \\ \boldsymbol{\zeta}_{q}\end{array}\right]$ is quasi-optimal

Thus feasibility, centrality and solution characteristics can be ascribed to pairs and points in a natural manner.

Lemma $8 \boldsymbol{\omega}=\left[\begin{array}{l}\boldsymbol{\xi} \\ \boldsymbol{\zeta}\end{array}\right]$ is a fixed-point $\Rightarrow$ (a) $\boldsymbol{\gamma}^{T} \boldsymbol{\omega}=0 ; \quad$ (b) $\Pi_{\boldsymbol{\gamma}} \boldsymbol{\gamma}=\mathbf{0}$

\section{Proof}

(a) $\boldsymbol{\omega}=\mathfrak{P} \boldsymbol{\omega} \Rightarrow \boldsymbol{\gamma}^{T} \boldsymbol{\omega}=\boldsymbol{\gamma}^{T} \mathfrak{P} \boldsymbol{\omega} \stackrel{(29 \mathrm{~b})}{\Rightarrow} \boldsymbol{\gamma}^{T} \boldsymbol{\omega}=\mathbf{0}^{T} \boldsymbol{\omega}=0$.

(b) $\boldsymbol{\gamma}^{T} \boldsymbol{\omega}=0 \Rightarrow \boldsymbol{\gamma}^{+} \boldsymbol{\omega}=0 \Rightarrow \boldsymbol{\gamma} \boldsymbol{\gamma}^{+} \boldsymbol{\omega}=\mathbf{0} \Rightarrow \Pi \boldsymbol{\gamma} \boldsymbol{\gamma}=\mathbf{0}$.

\subsubsection{The discriminant and centrality}

Definition $6 d(\boldsymbol{\omega})=-\boldsymbol{\beta}^{+} \boldsymbol{\omega}$ is called the discriminant of $\boldsymbol{\omega}$.

Lemma $9 \boldsymbol{\omega}$ is a central-point $\Rightarrow d(\boldsymbol{\omega})=\boldsymbol{\beta}^{+} \boldsymbol{\beta} \in\{0,1\}$.

$\operatorname{Proof} \boldsymbol{\omega}=\Pi_{\mathfrak{Z}} \boldsymbol{\omega}-\boldsymbol{\beta} \Rightarrow \boldsymbol{\beta}^{+} \boldsymbol{\omega}=\boldsymbol{\beta}^{+} \Pi_{\mathfrak{Z}} \boldsymbol{\omega}-\boldsymbol{\beta}^{+} \boldsymbol{\beta} \Rightarrow-\boldsymbol{\beta}^{+} \boldsymbol{\omega}=\boldsymbol{\beta}^{+} \boldsymbol{\beta} \Rightarrow d(\boldsymbol{\omega})=\boldsymbol{\beta}^{+} \boldsymbol{\beta}$.

Note that the functions $\mathfrak{C}_{1}$ and $\mathfrak{P}$ are idempotents which commute.

The following lemma shows that the discriminant determines precisely whether or not a fixed-point is central, and guarantees that applying $\mathfrak{C}_{2}$ to a fixed-point yields a central fixed-point:

Lemma $10 \boldsymbol{\omega}$ is a central fixed-point iff $\boldsymbol{\omega}$ is a fixed-point and $d(\boldsymbol{\omega}) \in\{0,1\}$. 
Proof The forward implication follows from Lemma 9; conversely,

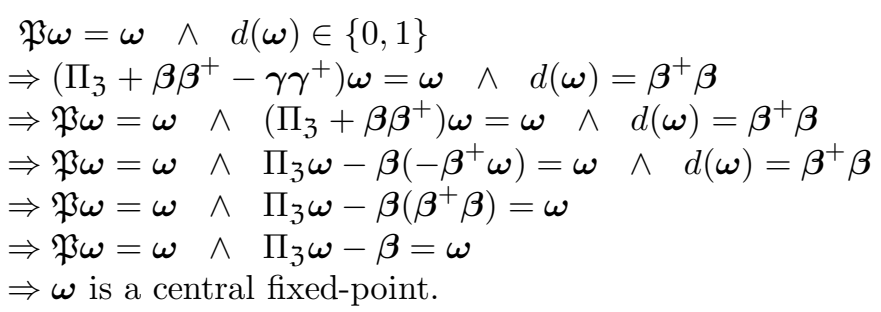

Consistent with Theorem 2 (c) and (d) in case (a), and with Lemma 10 in case (b), we define centralising functions:

\section{Definition 7}
(a) $\mathfrak{C}_{1}:\left[\begin{array}{l}\boldsymbol{\xi} \\ \boldsymbol{\zeta}\end{array}\right] \mapsto\left[\begin{array}{c}\mathfrak{A} \boldsymbol{\xi} \\ \mathfrak{D} \boldsymbol{\zeta}\end{array}\right]-\left[\begin{array}{l}\mathfrak{b} \\ \mathfrak{c}\end{array}\right]$ or
alternatively, $\mathfrak{C}: \boldsymbol{\omega} \mapsto \Pi_{\mathfrak{Z}} \boldsymbol{\omega}-\boldsymbol{\beta}$, and
(b) $\mathfrak{C}_{2}: \boldsymbol{\omega} \mapsto \boldsymbol{\omega} / d(\boldsymbol{\omega})$.

Lemma $11 \boldsymbol{\omega}$ is a central fixed-point iff $\boldsymbol{\omega}$ is a quasi-optimal central point.

Proof If $\boldsymbol{\omega}$ is a central fixed-point then by Lemma 8, $\boldsymbol{\omega}$ is quasi-optimal central. Conversely if $\boldsymbol{\omega}$ is quasi-optimal central, $\mathfrak{P} \boldsymbol{\omega}=\left(\Pi_{\mathfrak{Z}}+\Pi_{\boldsymbol{\beta}}-\Pi_{\gamma}\right) \boldsymbol{\omega} \stackrel{(\operatorname{Lemma} 8)}{=}\left(\Pi_{\mathfrak{Z}}+\Pi_{\boldsymbol{\beta}}\right) \boldsymbol{\omega}=\left(\Pi_{\mathfrak{Z}}+\right.$ $\left.\boldsymbol{\beta} \boldsymbol{\beta}^{+}\right) \boldsymbol{\omega}=\Pi_{\mathfrak{Z}} \boldsymbol{\omega}+\boldsymbol{\beta}\left(\boldsymbol{\beta}^{+} \boldsymbol{\omega}\right) \stackrel{(\text { Lemma } 9)}{=} \Pi_{\mathfrak{Z}} \boldsymbol{\omega}+\boldsymbol{\beta}\left(-\boldsymbol{\beta}^{+} \boldsymbol{\beta}\right)=\Pi_{\mathfrak{Z}} \boldsymbol{\omega}-\boldsymbol{\beta} \stackrel{\text { (Definition } 7)}{=} \mathfrak{C}_{1} \boldsymbol{\omega}=\boldsymbol{\omega}$, that is $\mathfrak{P} \boldsymbol{\omega}=\boldsymbol{\omega}$, so $\boldsymbol{\omega}$ is a central fixed-point.

So, $\boldsymbol{\omega}_{a}$ is an arbitrary non-negative central fixed-point $\stackrel{\operatorname{Lemma} 10}{\Rightarrow} \boldsymbol{\omega}_{c}=\boldsymbol{\omega}_{a} / d\left(\boldsymbol{\omega}_{a}\right)$ is a non-negative central fixed-point $\stackrel{\operatorname{Lemma}}{\Leftrightarrow} 11 \boldsymbol{\omega}_{c}$ is a non-negative quasi-optimal central point $(11)$

$\stackrel{(11)}{\Leftrightarrow} \boldsymbol{\omega}_{c}$ is a central solution point $\Leftrightarrow \mathfrak{N} \boldsymbol{\omega}_{c}$ is an invariant central solution pair. Thus we have the following theorem.

Theorem 4 The map $\mathfrak{N}:\left[\begin{array}{l}\boldsymbol{\xi} \\ \boldsymbol{\zeta}\end{array}\right] \mapsto(\boldsymbol{\xi}, \boldsymbol{\zeta})$ where $\boldsymbol{\xi}, \boldsymbol{\zeta} \in \Re^{m}$, with domain equal to the set of non-negative central fixed-points, and codomain equal to the set of invariant central solution pairs is a surjection.

\subsubsection{The unusual case}

There remains the unusual case of a zero central fixed-point. From Lemma 9, for central fixed-point $\boldsymbol{\omega}_{c f},-\boldsymbol{\beta}^{+} \boldsymbol{\omega}_{c f}=\boldsymbol{\beta}^{+} \boldsymbol{\beta} \in\{0,1\}$ and usually $-\boldsymbol{\beta}^{+} \boldsymbol{\omega}_{c f}=1$, however consider the unusual case where $-\boldsymbol{\beta}^{+} \boldsymbol{\omega}_{c}=0$, that is $\left[\boldsymbol{\omega}_{c f}=\Pi_{\mathfrak{Z}} \boldsymbol{\omega}_{c f}-\boldsymbol{\beta}\right] \wedge\left[\boldsymbol{\omega}_{c f}=\mathfrak{P} \boldsymbol{\omega}_{c f}\right] \wedge\left[-\boldsymbol{\beta}^{+} \boldsymbol{\omega}_{c f}=0\right]$

$\stackrel{(\operatorname{Lemma} 8)}{\Rightarrow}\left[\boldsymbol{\omega}_{c f}=\Pi_{\mathfrak{Z}} \boldsymbol{\omega}_{c f}-\boldsymbol{\beta}\right] \wedge\left[\boldsymbol{\omega}_{c f}=\mathfrak{P} \boldsymbol{\omega}_{c f}\right] \wedge\left[-\boldsymbol{\beta}^{+} \boldsymbol{\omega}_{c f}=0\right] \wedge\left[\boldsymbol{\gamma}^{+} \boldsymbol{\omega}_{c f}=0\right]$

$\Rightarrow\left[\boldsymbol{\omega}_{c f}=\Pi_{\mathfrak{Z}} \boldsymbol{\omega}_{c f}-\boldsymbol{\beta}\right] \wedge\left[\boldsymbol{\omega}_{c f}=\left(\Pi_{\mathfrak{Z}}+\boldsymbol{\beta} \boldsymbol{\beta}^{+}\right) \boldsymbol{\omega}_{c f}\right] \wedge\left[-\boldsymbol{\beta}^{+} \boldsymbol{\omega}_{c f}=0\right]$

$\Rightarrow\left[\boldsymbol{\omega}_{c f}=\Pi_{\mathfrak{Z}} \boldsymbol{\omega}_{c f}-\boldsymbol{\beta}\right] \wedge\left[\boldsymbol{\omega}_{c f}=\Pi_{\mathfrak{Z}} \boldsymbol{\omega}_{c f}\right] \Rightarrow \boldsymbol{\beta}=\mathbf{0}$. If $\boldsymbol{\beta}=\mathbf{0}$ then $\mathfrak{b}=\mathbf{0}$ and $\mathfrak{c}=\mathbf{0}$, and 
the invariant problems are $\max \left\{\mathbf{0}^{T} \mathfrak{x}: \mathfrak{A} \mathfrak{x} \geq \mathbf{0}\right\}$ and $\max \left\{\mathbf{0}^{T} \mathfrak{y}: \mathfrak{D} \mathfrak{y} \geq \mathbf{0}\right\}$, that is, find the solutions for the invariant problems $\{\mathfrak{x}: \mathfrak{A} \mathfrak{x} \geq \mathbf{0}\}$ and $\{\mathfrak{y}: \mathfrak{D} \mathfrak{y} \geq \mathbf{0}\}$ and the solutions to the original problems $\left\{A^{+}(\mathfrak{x}+\boldsymbol{b}): \mathfrak{A} \mathfrak{x} \geq \mathbf{0}\right\}$ and $\{\mathfrak{c}-\mathfrak{D} \mathfrak{y}: \mathfrak{D} \mathfrak{y} \geq \mathbf{0}\}$. So, under these

circumstances, every non-negative central solution fixed-point solution $\mathfrak{z}_{s}=\left[\begin{array}{l}\mathfrak{x}_{s} \\ \mathfrak{y}_{s}\end{array}\right]$ yields solutions $A^{+}\left(\mathfrak{x}_{s}+\boldsymbol{b}\right)$ and $\mathfrak{c}-\mathfrak{D} \mathfrak{y}_{s}$ to the original primal and dual respectively.

\section{Conclusion}

Summing up, the one-to-one relationship between the original and invariant problems is shown in the lower two-thirds of Figure 1, while the one-to-one relationship between the invariant and fixed-point problems is shown in the upper two-thirds of the figure. Thus the central original problems have been represented as the central invariant problems, and the central invariant problems have been represented as the central fixed-point problem; since the central problems encapsulate all solutions, this completes the representation of the LP problem aa a fixed-point problem.

\section{References}

[1] Pyle, L. D. The generalized inverse in linear programming - basic structure. SIAM Journal on Applied Mathematics. 23(3): 1972.

[2] Pyle, L. D. and Cline, R. E. The generalized inverse in linear programming - interior gradient projection methods. SIAM Journal on Applied Mathematics. 24(4): 1973.

[3] Cline, R. E. and Pyle, L . D. The generalized inverse in linear programming, an intersection projection method and the solution of a class of structured linear programming problems, SIAM Journal on Applied Mathematics, 1973. 24: 338-351.

[4] T.M. Nguyen, Applications of Generalized Inverse to Circulant Matrices, Intersection Projections, and Linear Programming. University of Houston: Ph. D. Thesis. 1982.

[5] Bruni, A. J. Nonnegative, Nontrivial Fixed Points of Orthogonal Projections. Department of Mathematics, University of Houston: Ph.D. Thesis. 1985.

[6] Albert, A. A. Regression and the Moore-Penrose Pseudoinverse. Houston: Academic Press. 1985.

[7] Clifford, A. H. and Preston, G. B. The Algebraic Theory of Semigroups, The American Mathematical Society, Volume 1. 1961.

[8] Abdullah, J. Fixed Point Algorithms for Linear Programming. Department of Economics, University of Birmingham, England: Ph.D. Thesis. May, 1992.

[9] Abdullah, J. Optimization by the Fixed-Point Method, Version 2.17, March, 2016, http://www.optimization-online.org/DB_HTML/2007/09/1775.html.

[10] Schrijver, A. Theory of Linear and Integer Programming. New York: Wiley. 1986. 